\title{
Elaboração de massa de pizza com teor de sódio reduzido e enriquecida com farinha de aveia
}

\section{Mass preparation of pizza with reduced sodium content and enriched with oatmeal}

\author{
Josefa Raniely Pereira de Sousa ${ }^{1 *}$, Jayuri Susy Fernandes de Araújo ${ }^{2}$, Inês Maria Barbosa Nunes Quiroga ${ }^{3}$, Mônica Correia \\ Gonçalves ${ }^{4}$, Mônica Tejo Cavalcanti ${ }^{4}$
}

\begin{abstract}
Resumo: Existe uma forte tendência pela busca da substituição de ingredientes que, quando ingeridos em excesso, causam danos à saúde. Preocupados com a saudabilidade, os consumidores exigem mudanças, substituições e enriquecimento aos alimentos para que tragam algum benefício ao organismo, além da função básica de nutrir, e que ainda o satisfaça sensorialmente. Portanto, o objetivo deste trabalho foi desenvolver massas para pizza com teor de sódio reduzido, através da substituição parcial de $\mathrm{NaCl}$ por $\mathrm{KCl}$, além de enriquecê-la com farinha de aveia, que tem benefícios comprovados à saúde, para verificar sua aceitação sensorial. Em uma análise sensorial preliminar constatou-se a aceitação da substituição de 50\% do $\mathrm{NaCl}$ por KCl. Utilizando esta formulação aceita como amostra padrão realizou-se a substituição parcial da farinha de trigo por farinha de aveia, nas proporções de 10 (F1), 15 (F2) e 20\% (F3). Os atributos sensoriais de sabor e aceitação global não diferiram estatisticamente entre si para as diferentes concentrações de farinha de aveia. Com relação a aparência, a amostra F2, com $15 \%$ de substituição, foi a única que apresentou diferença estatística significativa. Foi possível constatar, através das respostas de um questionário online, que os consumidores estão preocupados com os seus hábitos alimentares e pagariam a mais por produtos com algum tipo de substituição que beneficie sua saúde. A massa para pizza com teor de sódio reduzido e enriquecida com farinha de aveia foi bem aceita, principalmente na concentração relativa à 15\% de substituição.
\end{abstract}

Palavras-chave: Hábitos alimentares; Avena sativa L.; alimentos funcionais; análise sensorial.

\begin{abstract}
There is a strong tendency for the search for replacement ingredients that when ingested in excess, cause damage to health. Concerned about the healthiness, consumers demand changes, substitutions, and enriched foods to bring some benefit to the body, beyond the basic function of nurture and still satisfies sensuously. Therefore, the objective of this study was to develop pasta to pizza with reduced sodium content by partial replacement of $\mathrm{NaCl}$ by $\mathrm{KCl}$, and enrich it with oatmeal, which has proven health benefits, to check their sensory acceptance. In a primary sensory analysis it was found acceptance in replacement of $50 \%$ of $\mathrm{NaCl}$ for $\mathrm{KCl}$. Using this formulation accepted as a standard sample was carried out partial substitution of wheat flour by oatmeal, in the proportions of 10 (F1) 15 (F2) and 20\% (F3). The sensory attributes of taste and overall acceptance were not statistically different from each other for different oatmeal concentrations. Regarding the appearance, the sample F2, with $15 \%$ replacement, it was the only one that showed a statistically significant difference. It was found, through the answers to an online questionnaire, consumers are concerned about their eating habits and pay more for products with some kind of replacement that will benefit your health. The dough for pizza with low sodium content and enriched with oat flour was well accepted, particularly in the relative concentration of $15 \%$ substitution.
\end{abstract}

Key words: Eating habits; Avena sativa L.; functional foods; sensory analysis.

\footnotetext{
*Autor para correspondência

Recebido para publicação em 10/02/2016; aprovado em 20/05/2016

${ }^{1}$ Engenheira de Alimentos, Universidade Federal de Campina Grande (UFCG), Pombal-PB, E-mail: raniely.pereira@gmail.com

${ }^{2}$ Engenheira de Alimentos, UFCG, Pós-Graduanda em Qualidade e Segurança dos Alimentos, Faculdade SENAI da Paraíba, Sousa - PB; E-mail: jayuri.susy@gmail.com

${ }^{3}$ Mestre e Doutoranda em Ciência e Tecnologia de Alimentos - PPGCTA - UFPB. E-mail: inesnunes2503@hotmail.com

${ }^{4}$ Professora Mestre da Universidade Federal de Campina Grande, Pombal - PB, E-mail: mnygoncalves@ gmail.com

${ }^{5}$ Professora Doutora da Universidade Federal de Campina Grande, Pombal - PB, E-mail: monicatejoc@ yahoo.com.br
} 


\section{INTRODUÇÃO}

É crescente a preocupação dos consumidores com a alimentação saudável, mas a forma pela qual eles têm se alimentado não tem ajudado neste aspecto. A população procura demasiadamente por produtos práticos e prontos para o consumo, como os alimentos congelados, prontos ou semiprontos, além dos fast foods, que predominam nas grandes cidades (ABREU et al., 2001; MORAIS et al., 2015).

Em contrapartida ao ritmo de vida cada vez mais acelerado das pessoas e a comodidade que os alimentos prontos ou semiprontos trazem, e adequando-se a preocupação dos consumidores com a saúde, Vinhas (2011) afirma que a indústria tem se adaptado a estas necessidades, produzindo alimentos com teor de sódio reduzido, açúcar e gordura, como também adicionados de ingredientes que remeta algum apelo funcional.

A pizza é um produto que apresenta um alto índice de consumo, por ser um alimento prático, de sabor agradável e valor nutritivo, este último depende dos ingredientes utilizados tanto na massa quanto no recheio. Tal fato favorece a comercialização de pizzas em ambientes comerciais que não seja apenas pizzarias, como os supermercados, que oferecem o produto congelado e semipronto ao consumidor (PINHO; MACHADO; FURLONG, 2001).

O sódio é um nutriente essencial, porém, sua ingestão em excesso pode ocasionar o desenvolvimento de doenças crônicas não transmissíveis (DCNT), dentre elas a hipertensão, doenças cardiovasculares, osteoporose, acidente vascular cerebral e câncer (HE; MACGREGOR, 2009). O consumo do sódio está relacionado principalmente a ingestão de cloreto de sódio (mais conhecido como sal de cozinha), sendo este ingrediente adicionado aos alimentos durante as etapas de industrialização, no cozimento e à mesa dos consumidores. Adiciona-se o sal, principalmente, para conferir melhorias na textura, sabor e conservação dos alimentos (UK, 2008).

Uma das alternativa de substituição para o cloreto de sódio $(\mathrm{NaCl})$ mais comum é o cloreto de potássio $(\mathrm{KCl})$, para alimentos com o teor de sódio reduzido, para produtos comercializados e na alimentação oferecida em ambientes hospitalares (OLIVEIRA et al., 2013). Com propriedades correlatas ao $\mathrm{NaCl}$, o $\mathrm{KCl}$ é geralmente reconhecido como seguro (GRAS), e quando utilizado como substituto não perde sua funcionalidade (COLLINS, 1997). Muito embora, é necessário que seja considerado um inconveniente sobre o sabor, já que o $\mathrm{KCl}$ colabora com amargor e adstringência, não sendo facilmente disfarçado (CAUVAIN, 2007).

Além dos nutrientes habituais que os alimentos contém, podem ser agregados ingredientes que propiciem alguma vantagem à saúde, tais alimentos podem ser denominados como alimentos funcionais (MACIEL; PONTES; RODRIGUES, 2008), denominação que foi definida pelo Comitê de Alimentos e Nutrição do Instituto de Medicina (IOM, 1994). Ingredientes com tal função são muito importantes para a indústria de alimentos, podendo ser adicionados a produtos de panificação, tais como a pizza, em virtude da preocupação com a saúde dos consumidores (STRINGHETA et al., 2007).

A aveia (Avena sativa L.) é um cereal que tem destaque, quando comparado a outros cereais, em função do seu elevado valor nutricional, que se equilibra ao seu aporte energético, pois contém aminoácidos, ácidos graxos, vitaminas e sais minerais que são substanciais ao organismo humano e, sobretudo, pela composição de fibras alimentares, como a $\beta$-glucana, que é uma fibra solúvel conhecida por seus efeitos hipocolesterolêmicos comprovados (SÁ et al., 2000; DANIEL et al., 2006).

Para Frozza et al. (2002) os produtos de panificação fazem parte dos alimentos que têm sido utilizado para incluir fibras alimentares na dieta. Esse tipo de alimento é parte da dieta corriqueira da população, e a pizza está incluída, pois é um alimento frequentemente consumido pelas pessoas, em função do sabor, agilidade de preparo ou pelo fato de ser encontrado pronto ou semipronto.

É imprescindível para um alimento que além de seu valor nutritivo ele exerça satisfação e seja agradável ao consumidor, estes fatos são resultantes da harmonia entre diferentes atributos de qualidade sensorial, tais como, sabor, aparência, forma, textura, cor, odor, consistência e a interação entre diversos componentes, para que se consiga alcançar um equilíbrio total, que se reflita em qualidade elevada e boa aceitação por parte dos consumidores (TEIXEIRA, 2009).

Dentro desse contexto, vários trabalhos vêm desenvolvendo alternativas para alimentos tradicionais com teor de sódio reduzido, tais como os realizados por Nascimento et al. (2007) em salsichas, e Ignácio et al. (2013) em pão francês. A literatura apresenta ainda, diversas pesquisas com produtos de panificação que remeta algum apelo funcional (MACIEL; PONTES; RODRIGUES, 2008; BORGES et al., 2013;), incluindo o desenvolvimento de massas para pizza (FROZZA et al., 2002; RUSSO et al., 2012; COSTA et al., 2012; MORAIS et al., 2015).

Buscar uma alimentação apropriada e nutritiva é de suma importância, mas a satisfação sensorial também se faz necessária. Nota-se os esforços tanto da indústria quanto da comunidade científica para atender as demandas dos consumidores, que buscam cada vez mais por hábitos alimentares saudáveis. Neste sentido, objetivou-se avaliar o interesse dos consumidores quanto à alimentação saudável; elaborar massas para pizza com teor de sódio reduzido e enriquecidas com farinha de aveia e verificando sua aceitação sensorial.

\section{MATERIAL E MÉTODOS}

Com o intuito de investigar a real preocupação dos consumidores em relação aos hábitos alimentares foi realizado um questionário online (Google docs), com 150 pessoas, que esteve disponível na internet do dia 30 de agosto ao dia 10 de setembro.

Para a elaboração das massas para pizza, os ingredientes foram adquiridos em comércio local da cidade de Pombal, PB, Brasil e conduzidos para os Laboratórios de Tecnologia de Grãos e Cereais e de Análise sensorial do Centro de Ciência e Tecnologia Agroalimentar da Universidade Federal de Campina Grande.

Em um trabalho anterior, SOUZA et al., (2013) realizaram análise sensorial de massa de pizza substituída parcialmente por $\mathrm{KCl}$, com o intuito de verificar sua aceitação. Foram elaboradas três formulações, sendo uma utilizada como referência das massas de pizza comercializadas habitualmente, com adição total de $\mathrm{NaCl}$. Outra, sem adicionar nenhum dos sais $(\mathrm{NaCl}$ ou $\mathrm{KCl})$, a não ser o que foi proveniente dos demais ingredientes da formulação, e uma terceira com $50 \%$ de $\mathrm{NaCl}$ e $50 \%$ de $\mathrm{KCl}$. 
Constataram que esta última formulação apresentou aceitação sensorial satisfatória e não diferiu estatisticamente da massa convencional.

Deste modo, utilizou-se como formulação padrão (FP) para o presente trabalho, esta massa com substituição parcial de $\mathrm{NaCl}$ por $\mathrm{KCl}$, enriquecendo-a com farinha de aveia a partir da substituição parcial da farinha de trigo, nas proporções de $10(\mathrm{~F} 1), 15(\mathrm{~F} 2)$ e $20 \%(\mathrm{~F} 3)$, com relação ao peso total das farinhas adicionadas. Os ingredientes utilizados nas formulações das massas estão descritas na Tabela 1.

A princípio a água morna e o fermento biológico (levedura Saccharomyces cerevisiae) seco instantâneo (Dr.
Oetker) foram misturados e deixados em repouso durante 10 minutos. Logo após, adicionou-se o açúcar cristal (Parari), liteSalt (Linea), ovos, azeite de oliva (Gallo), margarina (Puro Sabor), farinha de trigo tradicional (Boa Sorte) e farinha de aveia (Quaker) nas proporções apresentadas na Tabela 1. Todos os ingredientes foram misturados e homogeneizados, e a massa obtida repousou por 40 minutos. Após o período de descanso, as massas foram abertas em forma circular com o auxílio de um rolo, para em seguida serem assadas em forno elétrico, pré aquecido durante 15 minutos, a temperatura de $200{ }^{\circ} \mathrm{C}$, por aproximadamente 20 minutos.

Tabela 1. Formulações das massas de pizza com teor de sódio reduzido e diferentes concentrações de farinha de aveia.

\begin{tabular}{|c|c|c|c|c|}
\hline \multirow{2}{*}{ Ingredientes $(\%)$} & \multicolumn{4}{|c|}{ Formulações } \\
\hline & FP & F1 & $\mathrm{F} 2$ & F3 \\
\hline Farinha de trigo & 57,1 & 51,4 & 48,6 & 45,7 \\
\hline Água & 28,6 & 28,6 & 28,6 & 28,6 \\
\hline Farinha de aveia & 0,0 & 5,7 & 8,6 & 11,4 \\
\hline Açúcar & 2,9 & 2,9 & 2,9 & 2,9 \\
\hline Azeite & 2,9 & 2,9 & 2,9 & 2,9 \\
\hline Margarina & 2,9 & 2,9 & 2,9 & 2,9 \\
\hline Ovo & 2,9 & 2,9 & 2,9 & 2,9 \\
\hline Fermento biológico & 1,7 & 1,7 & 1,7 & 1,7 \\
\hline LiteSalt* & 1,1 & 1,1 & 1,1 & 1,1 \\
\hline
\end{tabular}

* $13 \%$ de $\mathrm{NaCl}$ e $35 \%$ de $\mathrm{KCl}$ - produto comercial.

Para os testes sensoriais, foram recrutados 75 avaliadores não treinados com base nos hábitos alimentares e interesse em consumir pizza, estes, analisaram quatro amostras com relação à aceitação global, textura, aparência e sabor, de acordo com a escala hedônica de 9 pontos. Foi avaliada também a intenção de compra em uma escala de cinco pontos, variando de $5=$ Certamente compraria a $1=$ Certamente não compraria, e a ordem de preferência das amostras. (MEILGAARD; CIVILLE; CARR, 1999).

A análise sensorial foi conduzida em cabines individuais, com temperatura e iluminação controladas. As massas foram servidas no formato de pequenos discos uniformes (aproximadamente $10 \mathrm{~g}$ ), em pratos brancos descartáveis, codificados com números aleatórios de três dígitos em ordem de apresentação monádica e devidamente balanceada, acompanhados de copo com água e guardanapo de papel. (MACFIE al., 1989).

A análise estatística foi avaliada por análise de variância (ANOVA) e as médias comparadas pelo teste de Tukey a 5\% de significância, utilizando o programa estatístico Assistat versão 7.7 beta (2014).

\section{RESULTADOS E DISCUSSÃO}

Através da realização do questionário via internet constatou-se que das 150 pessoas que o responderam $74,5 \%$ não tem o hábito de seguir uma dieta equilibrada, e que a maioria $(62,4 \%)$ se preocupa com a quantidade de sal que ingere durante o dia. No entanto, as pessoas relataram ter dificuldade de encontrar alimentos processados com teor de sal reduzido (85,9\% dos entrevistados). Quando questionados sobre o hábito de consumir pizza, $60,4 \%$ deles afirmaram comer pizza uma vez por mês, seguido de $28,2 \%$ que comem duas vezes por semana e $11,4 \%$ uma ou mais vezes ao mês. A grande maioria dos consumidores $(90,5 \%)$ declarou não ter a opção de escolher o tipo de massa de pizza que quer consumir. Mas, se tivessem esta opção, $85,9 \%$ optariam por uma massa com reduzido teor de sal e $90,6 \%$ consumiriam uma massa com maior valor nutritivo que a massa convencional. Se fosse para pagar a mais por uma pizza com massa mais nutritiva e/ou com reduzido teor de sódio, 73,2\% dos consumidores pagariam.

A partir das avaliações sensoriais das massas para pizzas elaboradas, obteve-se os escores médios conforme apresentados na Tabela 2.

Tabela 2. Resultados da aceitação sensorial das massas de pizza com teor de sódio reduzido e diferentes concentrações de farinha de aveia.

\begin{tabular}{|c|c|c|c|c|}
\hline \multirow{2}{*}{ Atributos sensoriais } & \multicolumn{4}{|c|}{ Formulações* } \\
\hline & FP & $\mathrm{F} 1$ & $\mathrm{~F} 2$ & $\mathrm{~F} 3$ \\
\hline & $* * 6,64 \pm 1,38$ & & & \\
\hline Aparência & $\mathrm{a}^{* * *}$ & $6,63 \pm 1,74 \mathrm{a}$ & $5,89 \pm 1,72 b$ & $6,59 \pm 1,59 a$ \\
\hline Textura & $6,03 \pm 1,82 \mathrm{ab}$ & $5,63 \pm 1,89 \mathrm{~b}$ & $5,60 \pm 1,76 \mathrm{~b}$ & $6,57 \pm 1,79$ a \\
\hline Sabor & $6,4 \pm 1,53 \mathrm{a}$ & $6,23 \pm 1,73 \mathrm{a}$ & $5,83 \pm 1,46 a$ & $6,48 \pm 1,64 \mathrm{a}$ \\
\hline Impressão global & $6,16 \pm 1,85 \mathrm{a}$ & $6,01 \pm 1,70 \mathrm{a}$ & $5,92 \pm 1,55 \mathrm{a}$ & $6,07 \pm 1,94 \mathrm{a}$ \\
\hline
\end{tabular}


Os atributos sensoriais de sabor e impressão global não diferiram estatisticamente entre si para as diferentes concentrações de farinha de aveia aplicadas. Com relação a aparência, a amostra F2, com $15 \%$ de substituição, foi a única que apresentou diferença estatística significativa. Quanto a textura, a amostra padrão (FP) não diferiu das demais, e as amostras F1 e F2 não diferiram entre si, mas ambas apresentaram diferença com relação a amostra F3. A média dos escores para os parâmetros sensoriais avaliados ficou em torno de 6 (gostei ligeiramente), que de acordo com metodologia citada por Dutcosky (2007) com esse escore o alimento foi aceito.

A massa para pizza mais preferida entre os avaliadores foi a amostra F2 com 35\% de preferência, seguida da amostra FP (31\% de preferência), da amostra F1 (10\% de preferência), e por último, a menos citada como amostra preferida (apenas $9 \%$ ) foi a formulação $\mathrm{F} 3$, onde foi adicionada a maior quantidade de farinha de aveia, $20 \%$.

Costa et al. (2012) substituíram $20 \%$ de farinha de trigo por farinha da amêndoa da manga na elaboração de massa de pizza e obtiveram boa aceitação sensorial, assim como Morais et al. (2015) ao elaborarem massa para pizza com farinha de amêndoas da castanha de caju, e afirmaram que este tipo de substituição agrega um elevado valor nutricional a pizza, ao compará-la a pizza tradicional com adição de farinha de trigo. Ambos os autores dos trabalhos citados, sugerem a aplicação de tais substituições em outros produtos tais como, pães, bolos, esfihas, pasteis, entre outros.

Um outro estudo, realizado por Russo et al. (2012), quanto a aceitação, entre adolescentes, de massa para pizza enriquecida com farinha de trigo integral e farinha de linhaça, onde foram elaboradas 5 formulações, sendo uma padrão e as outras 4 com variações nas concentrações da farinha de linhaça de 2,5\%, 5,0\%, 7,5\% e 10\%, constataram que a adição de 5,0\% da farinha de linhaça obteve boa aceitação sensorial, sendo semelhante à apresentada pelo produto padrão.

A respeito da intenção de compra das massas elaboradas, a maioria dos avaliadores $(31 \%)$ afirmaram que certamente comprariam a amostra F2, constatando o que já foi verificado quanto a ordem de preferência por esta formulação. Apenas 7\% dos avaliadores afirmaram que certamente não comprariam a formulação F2. A formulação padrão foi a segunda que teve maior intenção de compra e a amostra F3 foi a que apresentou a menor intenção de compra, corroborando mais uma vez com os resultados verificados na preferência dos consumidores.

\section{CONCLUSÕES}

Os consumidores estão preocupados com os seus hábitos alimentares e pagariam a mais por produtos com algum tipo de substituição que beneficie sua saúde.

A massa para pizza com teor de sódio reduzido e enriquecida com farinha de aveia foi bem aceita, principalmente na concentração relativa à $15 \%$ de substituição.

\section{REFERÊNCIAS}

ABREU, E. S.; VIANA, I. C.; MORENO, R. B.; TORRES, E. A. F. S. Alimentação mundial - uma reflexão sobre a história. Saúde e Sociedade, São Paulo, v. 10, n. 3, p. 3-14, 2001.
BARBOZA, L. M. V.; FREITAS, R. J. S.; WASZCZYNSKYJ, N. Desenvolvimento de produtos e análise sensorial. Brasil Alimentos, Pinheiros, n. 18, 2003.

BORGES, J. T. S.; PIROZI, M. R.; VIDIGAL, J. G.; PAULA, C. D.; SILVA, N. A. S. Utilização de farinha mista de trigo e quinoa na elaboração de bolos. Revista Brasileira de Tecnologia Agroindustrial, v.7, n.2, p.1034-1048, 2013.

CAUVAIN, S. P. Reduced salt in bread and other baked products. In: KILCAST, D.; ANGUS, F. Reducing Salt in Foods. Boca Raton: CRC Press LLC. part 3, p. 283-295, 2007.

COLLINS, J. E. Reducing salt (sodium) levels in process meat poultry and fish products. In: PEARSON, A. M.; DUTSON, T. R. (Ed.) Advances in meat research: production and processing of healthy meat, poultry and fish products. London: Blackie Academic \& Professional, p. 283-297, 1997.

COSTA, T. S.; SILVA, V. C.; FERNANDES, V. S.; CAVALCANTI, M. T.; FlORENTINO, E. R. Avaliação sensorial de massa de pizza enriquecida com farinha do resíduo de extração do amido da amêndoa da manga (Mangifera indica L.) In: Encontro Nacional de Educação, Ciência e Tecnologia, UEPB, 2012, Campinha Grande. Anais... Disponível em: <http://www.editorarealize.com.br/revistas/enect/trabalhos/Po ster_275.pdf $>$. Acesso em: 18 set. 2016.

DANIEL, A. P.; BOCHI, V. C.; STEFFENS, C.; SILVA, L . P.; EMANUELLI, T. Fracionamento a seco da farinha de aveia e modificação química da fração rica em amido. Ciência e tecnologia de Alimentos, Campinas, v.26, p.936-943, 2006.

DUTCOSKY, S. D. Análise sensorial de alimentos. 2. ed. Curitiba: Champagnat, 2007, 239 p. ERTEKIN, B.; GUZELSEYDIM, Z. B. Effect of fat replacers on kefir quality. Journal of the Science of Food and Agriculture, n. 4, v. 90, p. 543-548, 2010.

FROZZA, J.; PENTEADO, T. P. S.; CAVASSIN, T. A.; BORGES, J. N. Pizza enriquecida com fibras para pessoas com diverticulose. Visão Acadêmica, Curitiba, v. 3, n. 2, p. 87-94, 2002.

HE F. J.; MACGREGOR G. A. A comprehensive review on salt and health and current experience of worldwide salt reduction programmes. Journal of Human Hypertension, v. 23, n. 6, p. 363-384, 2009.

IGNÁCIO, A. K. F.; RODRIGUES, J. T. D.; NIIZU, P. Y.; CHANG, Y. K. Efeito da substituição de cloreto de sódio por cloreto de potássio em pão francês. Brazilian Journal of Food Technology, Campinas, v.16, n.1, p.1-11, 2013.

INSTITUTE OF MEDICINE (IOM). How should the recommended dietary allowances be revised? food nutrition board. Washington, DC: National Academy, 1994. 46p.

MACFIE, H. J.; BRATCHELL， N.; GREENHOFF， K.; VALLIS, L. V. Designs to balance the effect of order of 
presentation and first-order carry-over effects in hall tests. Journal of Sensory Studies, Malden, v. 4, p. 129-148, 1989.

MACIEL, L. M. B.; PONTES, D. S.; RODRIGUES, M. C. P. Efeito da adição de farinha de linhaça no processamento de biscoito tipo cracker. Revista Alimentos e Nutrição, Araraquara, v. 19, n. 4, p. 385-392, 2008.

MEILGAARD, M.; CIVILLE, G. V.; CARR, B. T. Sensory evaluation techniques. 3rd ed. Boca Raton: CRC, 1999. 390p.

MORAIS, B. A.; LEMOS, L. S.; IUNES, M. F.; MIGUEL, S.; RODRIGUES, M. C. P. Elaboração e análise sensorial de massa de pizza com farinha de amêndoas da castanha de caju. In: Congresso Brasileiro de Engenharia Química. Anais... [= Blucher Chemical Engineering Proceedings, v. 1, n. 2, p. 3924-3930]. São Paulo: Blucher, 2015.

NASCIMENTO, R.; CAMPAGNOL, P. C. B.; MONTEIRO. E. S.; POLLONIO, M. A. R. Substituição de cloreto de sódio por cloreto de potássio: influência sobre as características físico-químicas e sensoriais de salsichas. Revista Alimentos e Nutrição, Araraquara, v.18, n.3, p.297-302, 2007.

OLIVEIRA, F. D; COELHO, A. R; BURGARDT, V. C. F; HASHIMOTO, E. H; LUNKES, A. M; MARCHI, J. F; TONIAL, I. B. Alternativas para um produto cárneo mais saudável: uma revisão. Brazilian Journal of Food Technology, Campinas, v.16, n.3, p.163-174, 2013.

PINHO, B. H. DA S.; MACHADO, M. I. F.; FURLONG, E. B. Propriedades fİsico-quİmicas das massas de pizza semiprontas e sua relação com o desenvolvimento de bolores e leveduras. Revista do Instituto Adolfo Lutz, São Paulo, v.60, n.1, p. 35-41, 2001.

RUSSO, C. B.; SOSTISSO, C. F.; PASQUAL, I. N.; NOVELLO, D.; DALLA SANTA, H. S.; BATISTA, M. G. Aceitabilidade sensorial de massa de pizza acrescida de farinhas de trigo integral e de linhaça (Linum usitatissimum
L.) entre adolescentes. Revista do Instituto Adolfo Lutz, São Paulo, vol. 71, n.3, p.488-494, 2012.

SILVA, F. A. S. ASSISTAT: Versão 7.7 beta. DEAG-CTRNUFCG. Atualizado em 04 de janeiro de 2016. Disponível em: $<$ http://www.assistat.com/>.

STRINGHETA, P. C.; OLIVEIRA T. T.; GOMES, R. C.; AMARAL, M. P. H.; CARVALHO, A. F.; VILELA, M. A. P. Políticas de saúde e alegações de propriedades funcionais e de saúde para alimentos no Brasil. Revista Brasileira de Ciências Farmacêuticas, São Paulo, v.43, n.2, p.181-194, 2007.

TEIXEIRA, L.V. Análise Sensorial na indústria de alimentos. Revista do Instituto de Laticínios Cândido Tostes, v. 64, n. 366, p. 12-21, 2009.

UK. Trading Standards Institute. The salt reduction toolkit. $2^{\mathrm{a}}$ edição, p. 33, 2008. Disponível em: < http://www.tradingstandards.uk/policy/policysaltreductiontoolkit.cfm >. Acesso em: 17 set. 2016.

VINHAS, A. D. M. Elaboração e avaliação sensorial de massa de pizza com adição de fibra de soja. 2011. 87f. Monografia (Graduação em Engenharia de Alimentos) Universidade Federal do Rio Grande do Sul, Porto Alegre. 2011.

SÁ, R. M.; DE FRANCISCO, A.; OGLIARI, P. J.; BERTOLDI, F. C. Variação no conteúdo de beta-glucanas em cultivares brasileiros de aveia. Ciência e Tecnologia de Alimentos, Campinas, v.20, n.1, p.99-102, 2000.

SOUZA, J. R. P.; CAVALCANTI, M. T.; COSTA, T. R. S.; SILVA, A. F.; MARQUES, I. R.; FERREIRA, W. C. Aceitação de massa de pizza com substituição parcial do cloreto de sódio por cloreto de potássio. In: Simpósio Latino Americano de Ciência dos Alimentos, 10., 2013, Campinas. Ciência de alimentos: impacto na nutrição e saúde: Anais... Campinas: Unicamp, 2013. 